Ann. Biol. anim. Bioch. Biophys., 1974, 14 (I), 27-39.

\title{
UTILISATION DE PROGESTAGENES EN IMPLANTS SOUS-CUTANÉS POUR LA MAÎTRISE DES CYCLES SEXUELS CHEZ LES BOVINS
}

\author{
D. CHUPIN, F. DELETANG*, M. PETIT*, J. PELOT, \\ F. LE PROVOST**, R. ORTAVANT, M. PAREZ* et P. MAULÉoN
}

\author{
Station de Physiologie de la Reproduction, \\ Centre de Recherches de Tours, I. N.R.A., \\ Nouzilly B.P. 1, 37380 Monnaie \\ * $U$. N.C.E. I. A., 149 rue de Bercy, \\ 75012 Paris \\ ** Lathevet (Département Searle), \\ 20 rue des Fossés St-Jacques \\ 75005 Paris
}

\section{RÉSUMÉ}

L'efficacité des implants sous-cutanés pour la maîtrise des cycles chez les bovins a été testées pour trois progestagènes (norethandrolone, $\mathrm{SC}$ 2 roog, FGA). Elle dépend de la dose initiaie de progestagène contenue dans l'implant et de la durée de séjour de celui-ci.

Un allongement de la durée de séjour diminue le taux d'inhibition de l'ostrus. Pour les traitements longs ( 6 ou I 8 jours), le degré de synchronisation (exprimé en pourcentage d'animaux en œstrus en quatre jours après le retrait) augmente avec la dose (de 6r,5 p. Ioo à 84,6 p. Ioo pour les implants SC 2 IoOg lorsque la dose passe de 6 à $\mathrm{r} 2 \mathrm{mg}$ ).

Une deuxième expérience réalisée dans des élevages privés a permis d'étudier les paramètres qui agissent sur la fertilité. La dose de progestagène a peu d'effets. Par contre, un allongement de la durée de séjour entraîne une baisse de la fertilité à l'ostrus induit $(49,4$ p. Ioo vs 27,3 p. 100 pour des traitements de io et I 6 jours respectivement avec des implants SC 2 Ioog).

L'injection de valérate d'cestradiol le jour de la pose de l'implant améliore la fertilité à l'œstrus induit indépendamment de la durée du traitement (implants SC 2 Ioog pendant I4 jours : $45,8 \mathrm{p}$. I oo avec valérate d'œestradiol vs $26,9 \mathrm{p}$. I oo sans cette injection).

L'emploi de progestagènes très actifs permet l'utilisation d'implants de petite taille. De plus, la technologie du support en hydron (p. I oo de liaisons croisées) doit être étudiée pour chaque progestagène (exemple : 4,8 p. Ioo pour le SC 2 I009). Des trois progestagènes que nous avons testés, seul le SC 2 roog est suffisamment actif pour être utilisé en implant. 
Plusieurs progestagènes : MAP, CAP (HANsEL, I966), MGA (ZimbelmaN, I964), DHPA (Wil,TBANK et al., I967), FGA (WISharT et Hoskin, I968; MAULEon et al., I968), SC 2I009 (WISHART, I972) noréthandrolone (LIANG et FosGATE, I97I) ont été utilisés pour maîtriser les cycles œstriens chez les femelles de races bovines.

Différentes voies d'administration du progestagène ont été utilisées :

- la voie orale (MAP, CAP, MGA, FGA, DHPA) pose des problèmes d'utilisation dans les élevages de type extensif,

- 1'injection (noréthandrolone, SC 2 Ioog, MGA, FGA) est peu pratique,

- la voie vaginale (FGA, MAP) pose parfois le problème de la rétention des éponges,

- l'implantation sous-cutanée (MGA, SC 2I0o9, noréthandrolone) a l'avantage d'être simple. Dans ce cas, l'un des problèmes posés est la taille de l'implant qui sera d'autant plus faible que le stéroïde utilisé aura une forte activité.

L'objet de cette étude est de tester trois progestagènes d'activités différentes (SC 2I009, FGA, noréthandrolone) incorporés dans des implants en polymétacrylate (Hydron).

Nous avons étudié l'importance de la nature et de la dose du progestagène utilisé, de la durée de séjour de l'implant et le rôle de l'injection de valérate d'œstradiol, qui, d'après WII,TBANK et KASSON (I968), permet de raccourcir la durée du traitement progestatif.

\section{MATÉRIEL ET MÉTHODES}

Nous avons utilisé des implants en polymétacrylate (Hydron laboratories) contenant l'un des trois progestagènes: SC 2 Ioo9, FGA, noréthandrolone (Searle et $\mathrm{C}^{\mathrm{o}}$ ).

Le polymétacrylate forme un réseau de mailles dont la densité est exprimée par le pourcentage de cross-link (ou taux de liaisons) qui représente le pourcentage de liaisons de type "pont" entre les chaînes linéaires de monometacrylate.

Les implants contenant du SC 2 Ioog ont été étudiés pour deux taux de cross-link (0,3 et 4,8 p. IOo). Les implants contenant du FGA et de la norethandrolone avaient un taux de crosslink de $\mathrm{I}, 2$ p. Ioo.

Pour les implants SC 2 roog la concentration en progestagène est constante par unité de longueur. Pour les implants FGA au contraire, la taille reste constante et la concentration varie.

Les implants ont été placés sous la peau, soit à la base de l'oreille pour ceux contenant du FGA ou du SC 2 roog, soit au niveau des fosses paralombaires pour ceux contenant la norethandrolone.

Les implants sont posés à l'aide d'un trocard et retirés après une légère incision du cuir. Pour les deux opérations, le matériel était désinfecté entre chaque vache.

Au retrait les implants ont été conservés pour doser la quantité restante de progestagène ( $\left.{ }^{1}\right)$.

Les détails du protocole expérimental sont portés dans le tableau I.

Tous les animaux traités pendant 9 , Io et $\mathrm{I} 2$ jours, ainsi qu'une partie des animaux traités pendant I 4 jours, ont reçu le jour de la pose de l'implant une injection intramusculaire de $5 \mathrm{mg}$ de valérate d' oestradiol.

Tous les animaux (excepté ceux avec les implants norethandrolone) reçurent en outre le jour du retrait de l'implant une injection intramusculaire de 800 U. I. de PMSG.

L'étude des paramètres de synchronisation a été réalisée en stations expérimentales. La détection d'cestrus était faite deux fois par jour, soit par passage d'un taureau vasectomisé, soit par observation du comportement des femelles entre elles.

(1) Dosages effectués par Wishart D. F. (G. D. Searle et Co., Angleterre). 
La fertilité a été étudiée sur divers troupeaux. Les animaux ont été inséminés systématiquement, à intervalles fixes par rapport au retrait de l'implant, sans observation de l'œstrus, Les schémas suivants ont été mis en place :
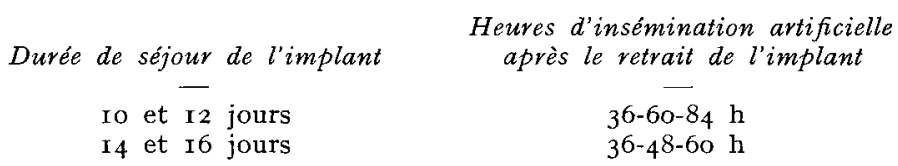

Les conditions de détection d'œstrus et le mode d'insémination artificielle des animaux non gestants après les I. A. systématiques varient en fonction des élevages et des régions où furent mises en place les expérimentations.

En particulier, le taureau, utilisé pour la fécondation des retours en cestrus a été introduit dans les troupeaux à des intervalles très variables (de quelques jours à plusieurs mois) après les I. A. systématiques.

\section{TABLEAU I}

\section{Protocoles expérimentaux}

\begin{tabular}{|c|c|c|c|c|}
\hline \multirow{2}{*}{ Progestagène } & \multirow{2}{*}{$\begin{array}{l}\text { P. } 100 \\
\text { cross-link }\end{array}$} & \multirow{2}{*}{ Doses et durées } & \multicolumn{2}{|c|}{ Paramètres étudiés } \\
\hline & & & Synchronisation & Fertilité \\
\hline \multirow{3}{*}{ SC 21009} & 0,3 & $\begin{array}{c}4,5 \text { et } 9 \mathrm{mg} \text { pendant } \\
9 \text { ou } 16 \mathrm{j}\end{array}$ & 48 génisses $F F P N$ & \\
\hline & & 6,12 et $18 \mathrm{mg}$ pendant & $\begin{array}{c}106 \text { vaches et génisses } \\
F F P N\end{array}$ & $\begin{array}{c}317 \text { vaches et génisses } \\
\text { Charolaises }\end{array}$ \\
\hline & 4,0 & $10,12,14,16 \mathrm{j}$ & 57 vaches Charolaises & $\begin{array}{c}448 \text { vaches et génisses } \\
\text { Salers, Aubrac }\end{array}$ \\
\hline FGA & 1,2 & $\begin{array}{l}50,100 \text { et } 150 \mathrm{mg} \\
\text { pendant } 10 \text { ou } 16 \mathrm{j}\end{array}$ & $\begin{array}{c}104 \text { vaches et génisses } \\
F F P N\end{array}$ & $\begin{array}{c}371 \text { vaches et génisses } \\
\text { Charolaises }\end{array}$ \\
\hline Noréthandrolone & 1,2 & $\begin{array}{c}250 \text { et } 500 \mathrm{mg} \text { pendant } \\
9 \text { ou } 18 \mathrm{j}\end{array}$ & $\begin{array}{c}81 \text { vaches et génisses } \\
F F P N\end{array}$ & \\
\hline
\end{tabular}

\section{Méthodologie de dépouillement}

Les paramètres de fertilité étudiés sont les suivants :

- la fertilité à l'œestrus induit : c'est le pourcentage d'animaux ayant mis bas (ou ayant eu un avortement constaté) consécutivement aux inséminations systématiques à l'œstrus induit ;

- le taux de mises-bas en un mois : c'est le pourcentage d'animaux ayant réellement mis bas avant un délai égal à $D_{G}+30$ jours après les inséminations systématiques $\left(\mathrm{D}_{\mathrm{G}}\right.$ : durée moyenne de gestation pour une race considérée). A ces animaux viennent s'ajouter ceux ayant avorté après les I. A. systématiques et les inséminations ou saillies après retour en œestrus dans les 30 jours qui suivent l'arrêt du traitement.

\section{RÉSULTATS}

\section{A. - Qualités de la synchronisation}

On a étudié le degré de l'inhibition de l'œestrus pendant le séjour de l'implant et la répartition des venues en œstrus après son retrait. 
I. Degré d'inhibition de l'aestrus.

Il peut être caractérisé, soit par le pourcentage d'animaux implantés ne manifestant pas d'œstrus (tab1. 2), soit par le pourcentage d'animaux venant en œestrus très rapidement après le retrait de l'implant (tabl. 4).

\section{TABLEAU 2}

Effrcacité des implants de progestagènes pour inhiber l'cestrus chez des génisses et des vaches de races FFPN et Charolaises en fonction de la nature et de la dose du progestagène et de la durée du traitement

(p. Ioo d'animaux ne manifestant pas d'œestrus pendant le séjour de l'implant) $\mathrm{XL}=$ "crosslinks" = pourcentage de liaisons

\begin{tabular}{|c|c|c|c|c|c|c|c|}
\hline \multirow[b]{2}{*}{ Produit } & \multirow{2}{*}{$\begin{array}{l}\text { Dose } \\
(\mathrm{mg})\end{array}$} & \multicolumn{6}{|c|}{ Durée du traitement } \\
\hline & & $\begin{array}{r}9-10 \\
(\end{array}$ & & 16 & & & urs \\
\hline SC 21009 XL 0,3 p. 100 & $\begin{array}{l}4,5 \\
9\end{array}$ & $\begin{array}{l}66,7 \\
75,0\end{array}$ & $\begin{array}{l}(12) \\
(12)\end{array}$ & $\begin{array}{l}18,2 \\
84,6\end{array}$ & $\begin{array}{l}(11) \\
(13)\end{array}$ & & \\
\hline SC 21009 XL 4,8 p. 100 & $\begin{array}{r}6 \\
12\end{array}$ & $\begin{array}{r}97,6 \\
100,0\end{array}$ & $\begin{array}{l}(83) \\
(22)\end{array}$ & $\begin{array}{l}84,6 \\
92,3\end{array}$ & $\begin{array}{l}(13) \\
(13)\end{array}$ & 85,7 & (28) \\
\hline FGA & $\begin{array}{r}50 \\
100 \\
150\end{array}$ & $\begin{array}{l}70,6 \\
72,3 \\
70,6\end{array}$ & $\begin{array}{l}(17) \\
(18) \\
(17)\end{array}$ & $\begin{array}{l}58,9 \\
61,2 \\
76,5\end{array}$ & $\begin{array}{l}(17) \\
(18) \\
(17)\end{array}$ & & \\
\hline Noréthandrolone & $\begin{array}{l}250 \\
500\end{array}$ & $\begin{array}{l}85,0 \\
95,0\end{array}$ & $\begin{array}{l}(20) \\
(20)\end{array}$ & & & $\begin{array}{l}45,0 \\
78,9\end{array}$ & $\begin{array}{l}(22) \\
(19)\end{array}$ \\
\hline
\end{tabular}

( ) Nombre d'animaux traités.

On observe un effet de la dose initiale de progestagène et de la durée de séjour de l'implant.

Lorsque la durée du séjour augmente le pourcentage d'animaux ne manifestant pas d'œstrus pendant le traitement diminue : pour des implants SC 2 Ioog (I2 mg) et pour des durées de Io jours, I 6 jours et $\mathrm{I} 8$ jours, ce pourcentage est respectivement de IOO,O p. IOO, 92,3 p. IOO, 85,7 p. IOO ; pour les implants de noréthandrolone (500 $\mathrm{mg}$ ) et pour des durées de Io et I 8 jours, il est de 95,0 p. Ioo et 78,9 p. Ioo. De plus, le pourcentage d'animaux venant en œstrus 24 heures après le retrait de l'implant augmente (pour les mêmes exemples que précédemment, il est respectivement de $7,6 \mathrm{p}$. Ioo, 36,3 p. Ioo et 54,4 p. Ioo pour le SC 2 roog et de o p. Ioo et 22,3 p. roo pour la noréthandrolone).

Pour une même durée de présence de l'implant, l'inhibition de l'œestrus est d'autant plus générale que la dose initiale de progestagène est plus élevée (implants de SC 2Ioog, à $0,3 \mathrm{p}$. Ioo de crosslink : $43,5 \mathrm{p}$. Ioo pour $4,5 \mathrm{mg}$ vs $80,0 \mathrm{p}$. Ioo pour $9 \mathrm{mg}$; implants de noréthandrolone : $64,3 \mathrm{p}$. Ioo pour $250 \mathrm{mg}$ vs $87,2 \mathrm{p}$. Ioo pour $500 \mathrm{mg}$ ). 
Ces variations en fonction de la dose initiale de progestagène et de la durée de séjour de l'implant sont d'autant plus nettes que le taux de crosslink du polymère (représentant le pourcentage de liaison de type " pont " entre les chaînes moléculaires) diminue.

Enfin, on constate une différence entre les produits : les implants contenant du FGA donnent un taux d'inhibition plus faible, pour toutes les doses et toutes les durées.

\section{Répartition des venues en astrus après le retrait des implants.}

Le pourcentage d'animaux venant en œestrus en quatre jours après le retrait des implants est plus élevé après un traitement de 16 jours qu'après un traitement de Io jours (tabl. 3) : 80,4 p. I00 vs 69,6 p. Ioo.

\section{TABLEAU 3}

Efficacité des implants de progestagènes pour synchroniser l'astrus

chez des génisses et des vaches de races FFPN et Charolaises en fonction de la nature et de la dose du progestagène et de la durée du traitement

(p. Ioo d'animaux en cstrus dans les 4 jours suivant la fin du traitement)

\begin{tabular}{|c|c|c|c|c|c|c|c|}
\hline \multirow[b]{2}{*}{ Produit } & \multirow[b]{2}{*}{$\begin{array}{l}\text { Dose } \\
\text { (mg) }\end{array}$} & \multicolumn{6}{|c|}{ Durée du traitement } \\
\hline & & \multicolumn{2}{|c|}{$\begin{array}{c}9-10 \text { jours } \\
(\%)\end{array}$} & \multicolumn{2}{|c|}{$\begin{array}{c}16 \text { jours } \\
(\%)\end{array}$} & \multicolumn{2}{|c|}{$\begin{array}{c}18 \text { jours } \\
(\%)\end{array}$} \\
\hline SC 21009 XL 0,3 p. 100 & $\begin{array}{l}4,5 \\
9\end{array}$ & $\begin{array}{r}83,3 \\
100,0\end{array}$ & $\begin{array}{l}(12) \\
(12)\end{array}$ & $\begin{array}{l}81,8 \\
92,3\end{array}$ & $\begin{array}{l}(11) \\
(13)\end{array}$ & & \\
\hline SC 21009 XL 4,8 p. 100 & $\begin{array}{r}6 \\
12\end{array}$ & $\begin{array}{l}68,7 \\
59,1\end{array}$ & $\begin{array}{l}(83) \\
(22)\end{array}$ & $\begin{array}{l}61,5 \\
84,6\end{array}$ & $\begin{array}{l}(13) \\
(13)\end{array}$ & 78,6 & (28) \\
\hline FGA & $\begin{array}{r}50 \\
100 \\
150\end{array}$ & $\begin{array}{l}58,8 \\
72,2 \\
64,7\end{array}$ & $\begin{array}{l}(17) \\
(18) \\
(17)\end{array}$ & $\begin{array}{l}52,9 \\
94,4 \\
82,4\end{array}$ & $\begin{array}{l}(17) \\
(18) \\
(17)\end{array}$ & & \\
\hline Noréthandrolone & $\begin{array}{l}250 \\
500\end{array}$ & $\begin{array}{l}35,0 \\
60,0\end{array}$ & $\begin{array}{l}(20) \\
(20)\end{array}$ & & & $\begin{array}{l}50,0 \\
68,4\end{array}$ & $\begin{array}{l}(22) \\
(19)\end{array}$ \\
\hline
\end{tabular}

( ) Nombre d'animaux traités.

Lorsque l'implant a été laissé en place pendant I 6 ou I 8 jours, ce pourcentage augmente avec la dose initiale de progestagène (de $6 \mathrm{r}, 5$ à 84,6 p. Ioo pour les implants de SC 2 Ioog lorsque la dose passe de 6 à I $2 \mathrm{mg}$, de 50,0 à 68,4 p. Ioo pour les implants de noréthandrolone lorsque la dose passe de 250 à $500 \mathrm{mg}$ ).

Lorsque l'implant a été laissé en place pendant Io jours on n'observe pas de différences systématiques entre les doses pour ce critère, exception faite des implants de noréthandrolone. 


\section{TABLEAU 4}

Pourcentages d'animaux en cestrus dans les 24 heures suivant le retrait de l'implant (par rapport aux animaux en cestrus en 4 jours)

en fonction de la dose et de la nature du progestagène et de la durée du traitement chez des génisses et des vaches de races FFPN et Charolaises

\begin{tabular}{|c|c|c|c|c|c|c|c|}
\hline \multirow{2}{*}{ Produit } & \multirow{2}{*}{$\begin{array}{l}\text { Dose } \\
\text { (mg) }\end{array}$} & \multicolumn{6}{|c|}{ Durée du traitement } \\
\hline & & $9-10$ & & 16 & & & urs \\
\hline SC 21009 XL 0,3 p. 100 & $\begin{array}{l}4,5 \\
9\end{array}$ & $\begin{array}{l}70,0 \\
25,0\end{array}$ & $\begin{array}{l}(10) \\
(12)\end{array}$ & $\begin{array}{r}88,9 \\
100,0\end{array}$ & $\begin{array}{r}(9) \\
(12)\end{array}$ & & \\
\hline SC 21009 XL 4,8 p. 100 & $\begin{array}{r}6 \\
12\end{array}$ & $\begin{array}{r}12,2 \\
7,6\end{array}$ & $\begin{array}{l}(57) \\
(13)\end{array}$ & $\begin{array}{l}37,3 \\
36,3\end{array}$ & $\begin{array}{r}(8) \\
(11)\end{array}$ & 54,4 & $(22)$ \\
\hline FGA & $\begin{array}{r}50 \\
100 \\
150\end{array}$ & $\begin{array}{l}20,0 \\
23,0 \\
36,3\end{array}$ & $\begin{array}{l}(10) \\
(13) \\
(11)\end{array}$ & $\begin{array}{l}33,3 \\
29,3 \\
28,5\end{array}$ & $\begin{array}{r}(9) \\
(17) \\
(14)\end{array}$ & & \\
\hline Noréthandrolone & $\begin{array}{l}250 \\
500\end{array}$ & $\begin{array}{c}28,6 \\
0\end{array}$ & $\begin{array}{r}(7) \\
(12)\end{array}$ & & & $\begin{array}{l}55,4 \\
22,9\end{array}$ & $\begin{array}{l}(11) \\
(13)\end{array}$ \\
\hline
\end{tabular}

( ) Nombre d'animaux traités.

TABLEAU 5

Infuence de la durée du traitement progestatif (SC 210o9) et de l'injection de valérate d'astradiol sur la fertilité à l'cestrus induit après vetrait des implants

\begin{tabular}{|c|c|c|c|c|c|}
\hline \multirow{3}{*}{ Types de traitements } & \multicolumn{4}{|c|}{ Races } & \multirow{3}{*}{ Total } \\
\hline & \multicolumn{2}{|c|}{ Salers-Aubrac } & \multicolumn{2}{|c|}{ Charolaise } & \\
\hline & $\begin{array}{l}\text { Génisses } \\
(\%)\end{array}$ & $\begin{array}{c}\text { Vaches } \\
(\%)\end{array}$ & $\begin{array}{c}\text { Génisses } \\
(\%)\end{array}$ & $\begin{array}{c}\text { Vaches } \\
(\%)\end{array}$ & \\
\hline $\begin{array}{c}\text { 10-12 j + valérate } \\
\text { d'œstradiol }\end{array}$ & $57,1 \quad(49)$ & $56,2 \quad(112)$ & $41,9 \quad(62)$ & $36,5 \quad(52)$ & $49,4 \quad(275)$ \\
\hline $\begin{array}{c}\text { 14-16 j sans valérate } \\
\text { d'oestradiol }\end{array}$ & $56,2 \quad(16)$ & $26,0 \quad(150)$ & $26,9 \quad(63)$ & $23,3 \quad(60)$ & $27,3 \quad(289)$ \\
\hline
\end{tabular}

( ) Nombre d'animaux traités. 


\section{B. - Fertilité}

I. Fertilité à l'oestrus induit.

On observe globalement que la fertilité à l'œestrus induit des animaux ayant reçu un implant pendant IO-I 2 jours avec injection de valérate d'œstradiol est supérieure à celle des animaux ayant reçu un implant pendant I4-I6 jours $(P<0,0 \mathrm{I})$ (tabl. 5).

\section{TABLEAU 6}

Influence du valérate d'estradiol sur la fertilité à l'ostrus induit après retrait d'implants contenant du SC 21009

(Durée du traitement : I4 jours)

(Vaches et génisses toutes races)

\begin{tabular}{l|rr}
\hline & P. 100 mises-bas à l'œstrus induit \\
Avec valérate d'œstradiol & 45,8 & $(194)$ \\
Sans valérate d'œstradiol & 26,9 & $(130)$ \\
\hline
\end{tabular}

Par ailleurs, pour une durée de traitement identique (I4 jours) (tabl. 6), la fertilité à l'œstrus induit pour les animaux ayant reçu une injection de valérate d'œstradiol est supérieure à celle des animaux n'ayant pas reçu cette injection (45,8 p. Ioo vs 26,9 p. $100=\mathrm{P}<0,05)$.

Enfin, globalement on ne constate pas d'influence de la dose initiale de progestagène sur la fertilité à 1'œstrus induit (tabl. 7).

\section{Taux de mises-bas en 30 jours.}

Le taux de mises-bas en 30 jours est plus élevé pour les animaux ayant reçu l'implant pendant une durée de IO-I2 jours avec injection de valérate d'œstradiol que chez les autres (tab1. 8) :

- Implants SC 2 I009 : IO-I2 j + V. E. vs I4-I6 j, respectivement 62,9 vs 5I,2 p. I0O;

- Implants FGA : Io j + V. E. vs I6 j, respectivement 57 vs 43 p. Ioo.

Cependant, cette différence est moins prononcée qu'à l'œstrus induit pour les implants contenant du SC 2 Ioog.

\section{DISCUSSION}

Les résultats inđiquent que l'efficacité des implants sous-cutanés dépend de trois paramètres :

- la dose initiale de stéroïdes contenue dans l'implant modulée par la possibilité de libération de ces stéroïdes, 
- la durée de séjour de l'implant,

- l'injection de valérate d'œstradiol lors de la pose de l'implant.

\section{TABLEAU 7}

Infiuence de la dose de SC 21009 contenu dans l'implant sur la fertilité à l'astrus induit

\begin{tabular}{|c|c|c|c|}
\hline \multirow{2}{*}{ Traitement } & \multicolumn{3}{|c|}{ Dose } \\
\hline & $\begin{array}{c}6 \mathrm{mg} \\
(\%)\end{array}$ & $\begin{array}{c}12 \mathrm{mg} \\
(\%)\end{array}$ & $\begin{array}{c}18 \mathrm{mg} \\
(\%)\end{array}$ \\
\hline $\begin{array}{c}10-12 \mathrm{j}+\text { valérate } \\
\text { d'œstradiol }\end{array}$ & $45,2 \quad(93)$ & $50,5 \quad$ & $52,9 \quad(85)$ \\
\hline $\begin{array}{c}14-16 \mathrm{j} \text { sans valérate } \\
\text { d'œstradiol }\end{array}$ & $27,6 \quad(98)$ & $27,0 \quad(100)$ & $27,5 \quad(91)$ \\
\hline
\end{tabular}

( ) Nombre d'animaux traités.

\section{TABLEAU 8}

Infuence de la durée du traitement et de l'injection de valérate d'astradiol sur le taux de mise-bas en 30 jours après cestrus induit par des implants contenant du SC 21009

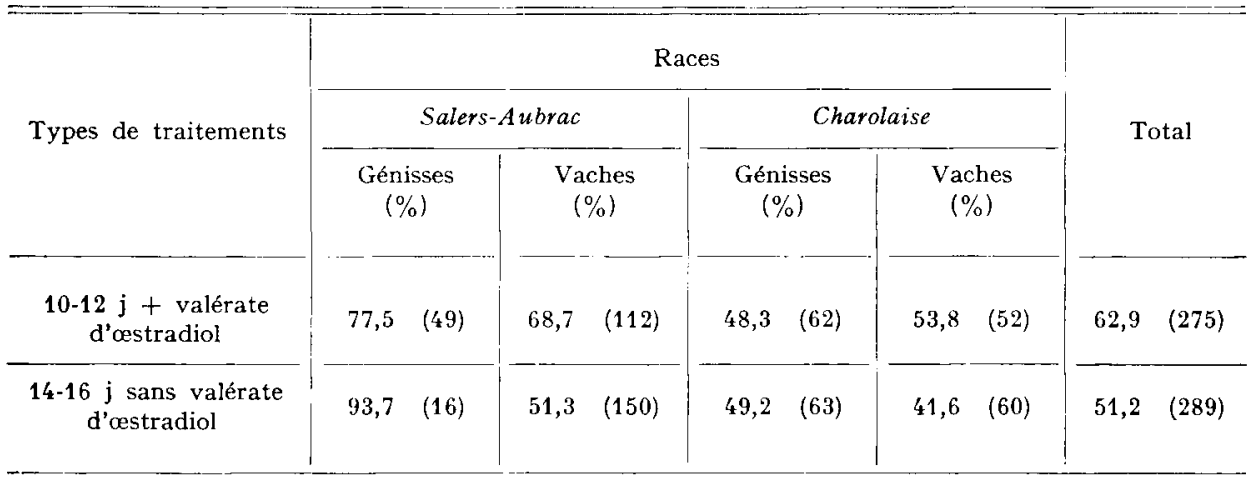

( ) Nombre d'animaux traités.

A. - Effet de la dose et de la durée de séjour des progestagènes et du taux de liaison du support en Hydron

L'élévation de la dose initiale de progestagène (SC 2 Ioog ou noréthandrolone) augmente le taux d'inhibition de l'œstrus et le taux de femelles synchronisées en quatre jours et diminue le pourcentage d'animaux en œstrus dans les 24 heures suivant le retrait de l'implant (animaux à la limite de la levée d'inhibition en fin de traitement). 
Pour un progestagène donné (SC 2Ioog), il existe un taux de liaisons optimum :

- Inhibition de l'œstrus et pourcentage des animaux en œestrus les premières 24 heures sont améliorés quand le taux de liaisons passe de $0,3 \mathrm{p}$. Ioo à 4,8 p. roo.

- Pour une dose initiale et une pourcentage de liaisons donné (I2 mg - SC 2roog, cross-link 4,8 p. IOo), le taux d'inhibition diminue lorsque la durée d'implantation augmente tandis que le pourcentage d'animaux en cestrus dans les premières 24 heures s'accroît.

Pour les implants de FGA, la dose initiale ne semble pas avoir d'influence sur les différents paramètres analysés.

Cet ensemble de faits montre l'importance des quantités libérées chaque jour, quantités qui sont dépendantes de la dose initiale, du pourcentage de liaisons et de la durée de séjour de l'implant.

Les dosages des quantités restantes effectués sur quelques implants après le retrait ont permis de déterminer les quantités moyennes de progestagènes qui avaient été utilisées par l'animal au cours du traitement. Les résultats ont été les suivants:

\begin{tabular}{|c|c|c|c|}
\hline \multirow{2}{*}{$\begin{array}{l}\text { Nature du } \\
\text { progestagène }\end{array}$} & \multirow{2}{*}{$\begin{array}{l}\text { Dose } \\
(\mathrm{mg})\end{array}$} & \multicolumn{2}{|c|}{$\begin{array}{l}\text { Quantités moyennes } \\
\text { disponibles par jour (mg) }\end{array}$} \\
\hline & & de $J_{0} \grave{a} J_{10}$ & de $\mathrm{J}_{10} \grave{\mathrm{a}} \mathrm{J}_{16}$ \\
\hline SC 21009 & $\begin{array}{r}6 \\
12 \\
18\end{array}$ & $\begin{array}{l}0,25 \\
0,52 \\
0,88\end{array}$ & $\begin{array}{l}0,11 \\
0,21 \\
0,31\end{array}$ \\
\hline FGA & $\begin{array}{c}150 \\
100 \\
50\end{array}$ & $\begin{array}{l}2,36 \\
2,25 \\
2,04\end{array}$ & $\begin{array}{l}0,43 \\
1,36 \\
0,66 i\end{array}$ \\
\hline
\end{tabular}

La quantité journalière de progestagène disponible par animal diminue du jour de pose au jour de retrait de l'implant. Elle semble proportionnelle à la dose initiale de progestagène pour les implants SC 2 Ioog et indépendante de ceile-ci pour les implants FGA.

En fait, il est difficile de distinguer la part de la dose initiale de celle de la surface de l'implant (Roseman et Higuchi, IO70; KINCL et RUDEL, I97I).

Le taux de liaisons peut aussi modifier les quantités journalières de progestagène disponible.

Ainsi, l'utilisation d'implants SC 2 Ioog avec un taux de liaisons de 0,3 p. Ioo entraîne une faible inhibition de l'œstrus et un fort pourcentage d'animaux venant en ostrus dans les 24 heures après le retrait de l'implant. Ceci est d'autant plus net que la durée du traitement est plus longue et peut expliquer une libération trop rapide du stéroïde dans l'organisme en début de traitement. Inversement, une faible libération quotidienne de FGA peut être imputée à un taux de liaisons trop élevé. Bien que les implants permettent d'obtenir un effet pharmacologique à des doses plus faibles que les injections huileuses (KINCL et RUDEL, I97I), les résultats de WISHART (I972) obtenus avec cette dernière technique (0,I4 $\mathrm{mg}$ et $2,4 \mathrm{mg}$ pour le SC 2 Ioog et le FGA respectivement) donnent une indication de ces doses minimums efficaces. 
On s'aperçoit alors que, pour le FGA, les quantités disponibles durant les derniers jours de traitements sont inférieures aux doses minimum efficaces.

Enfin, il faut noter que les mauvais résuitats obtenus avec les implants de FGA sont dus à des problèmes de technologie de l'implant puisque ce progestagène permet d'obtenir des taux de fertilité très satisfaisants par d'autres voies (injectable : WishaRT (I972), MARTIN et al., (I972) ; orale : CHUPIN et al., à paraître).

\section{B. - Effets de l'injection de valérate d'cestradiol}

\section{Fertilité à l'ostrus induit.}

Les animaux traités pendant ro et I 2 jours et une partie des animaux traités pendant $\mathrm{I}_{4}$ jours ont reçu le jour de la pose de l'implant une injection de $5 \mathrm{mg}$ de valérate d'œstradiol, ceci pour tenir compte des conclusions de WILTBANK et KaSSON (I968), selon lesquelles le valérate d'œestradiol possède une activité lutéolytique qui peut être utilisée pour raccourcir la durée d'un traitement de maitrise du cycle œstrien par les progestagènes. Malgré une inhibition plus efficace qu'en traitement long, le nombre d'animaux synchronisés en 4 jours est plus faible que dans ce dernier cas (pour les fortes doses de progestagènes). De plus, l'examen des dates d'œestrus avant et après un tel traitement montre que les animaux traités en début de cycles œstriens conservent leur cycle et viennent en œstrus à la date prévue.

Mais l'hypothèse de ces auteurs se révèle inexacte car l'injection de ro $\mathrm{mg}$ de valérate d'œstradiol le $6^{\mathrm{e}}$ jour du cycle (Bosc et al., communication personnelle) ne raccourcit pas la durée de celui-ci et la courbe de progestérone reste normale pendant un traitement avec noréthandrolone et injection de valérate d'œstradiol chez des animaux traités entre $J_{10}$ et $J_{16}$, la régression ne se produisant qu'à $J_{16}-J_{18}$ comme au cours d'un cycle normal (LEMON, communication personnelle). L'efficacité du valérate d'œstradiol comme facteur lutéolytique est donc très contestée. Pourtant les résultats de fertilité à l'œstrus induit après inséminations systématiques sont significativement plus élevés pour les traitements qui comportent une injection de valérate d'œestradiol. WiLtBank et al., (I97I) avec des implants de noréthandrolone trouvent un résultat semblable (6I p. Ioo vs 38 p. Ioo pour des traitements de 9 jours + valérate d'œstradiol ou de 16 jours respectivement). KNox et al. (I972) obtiennent 56 p. Ioo d'animaux gestants avec des implants SC 2 roog laissés en place pendant 9 jours (avec injection de $5 \mathrm{mg}$ de valérate d'œstradiol le jour de la pose), mais cet auteur n'indique pas de lot sans valérate.

Plusieurs hypothèses peuvent être proposées pour expliquer cet effet sur la fertilité :

- Le valérate d'œstradiol, qui a une action retard, peut modifier les conditions utérines et cervicales au moment de l'œstrus induit, si ces conditions sont défavorables par suite d'un excès de progestatif. Toutefois, HACkE'TT et al. (I972) trouvent lors de l'œstrus induit par un traitement de MAP que les taux d'œstrone et d'œstradiol sont plusieurs fois supérieurs à ce qu'ils observent lors de l'œstrus normal.

- Le valérate d'œestradiol peut agir sur la décharge des hormones gonadotropes. Les progestagènes utilisés inhibent la décharge de LH mais modifient peu les taux de FSH circulant. Ainsi, PRIEdkalns (I97I) a montré que, pendant un traitement 
avec MGA, la croissance folliculaire continue. Si le valérate d'œestradiol inhibe la décharge de FSH, la croissance folliculaire ne reprend qu'en fin de traitement comme dans un cycle normal.

On sait par ailleurs (Cumming et al., I970; Pelietien et Thimonier, I972) qu'après un traitement avec un progestagène la décharge de LH est, d'une part déplacée par rapport au début de l'œstrus et, d'autre part, d'un niveau moindre. De plus, chez la brebis, 1'injection d'un ostrogène (PELLETIER et ThIMONIER, Ig69) est suivie d'une décharge de $\mathrm{L} H$. On peut penser que le valérate d'œstradiol peut par son action retard améliorer la chronologie et l'amplitude des décharges hypophysaires.

- Le valérate d'œstradiol peut agir au niveau des récepteurs ovariens pour augmenter leur sensibilité aux hormones gonadotropes (GOLDENBERG et al., I972). La réponse ovarienne aux décharges hypophysaires en fin de traitement serait améliorée même si ces décharges sont d'un niveau inférieur à celles reportées lors d'un œestrus normal.

Il est difficile, pour l'instant, d'estimer la valeur de ces différentes hypothèses.

\section{Taux de mise-bas en 30 jours.}

L'amélioration de la fertilité observée à l'œstrus induit en usant de l'injection de valérate n'apparaît pas aussi nettement pour le taux de mises-bas en 30 jours. De plus, le taux d'animaux fécondés au cours de la période entre l'œstrus induit et 30 jours est faible (en moyenne $30 \mathrm{p}$. Ioo des animaux non fécondés à l'œstrus induit). On peut supposer qu'il existe un problème au cours de cette période, soit du fait de retours en œstrus irréguliers, soit du fait d'une mauvaise fertilité au deuxième œstrus. Ceci semble peu probable car les essais réalisés en inséminant uniquement au second œstrus sans traitement indiquent une fertilité normale (CHUPIN et al., à paraître) Ces résultats de fertilité ont été obtenus dans des zones d'élevage extensif, et les mauvaises conditions de détection d'œstrus ne permettent pas d'établir une courbe des retours en œestrus. Plusieurs hypothèses peuvent être proposées.

Tout d'abord, la faible fertilité après un traitement sans valérate d'œestradiol peut expliquer en elle-même un taux plus élevé de fécondation au I er retour en œstrus puisqu'un nombre plus élevé d'animaux a la possibilité de présenter un retour dans une période de 30 jours. On peut toutefois penser également que le valérate d'œstradiol modifie la distribution des retours en œstrus. En effet, si Bosc et al. (communication personnelle) n'observent pas d'effet de l'injection de Io mg de valérate d'œstradiol sur le cycle du traitement, ils notent par contre 30 p. Ioo de cycles courts (3 à Io jours) pour le cycle suivant. De même après un traitement avec la noréthandrolone pendant ro jours avec injection de valérate d'œestradiol le premier jour du traitement, nous avons observé ro $p$. Ioo de retours en œstrus tardifs (de 35 à 67 jours). Dans ces conditions, un certain nombre d'animaux, soit ne reviendraient pas en cestrus dans les 30 jours suivant le traitement, soit reviendraient rapidement après l'œstrus induit à une époque où l'éleveur observe moins attentivement les retours. Dans l'un et l'autre cas, ils ne seraient pas fécondés dans la période de 30 jours après le retrait de l'implant.

On constate en fait que les taux de fécondation sur le premier retour dans les $3^{\circ}$ jours qui suivent le retrait sont comparables qu'il y ait ou non injection de valérate 
d'œstradiol (28 p. Ioo avec valérate d'œestradiol). Ce taux s'explique en grande partie par les mauvaises conditions de détection d'œstrus rencontrées dans les élevages de type extensif. Dans ces conditions, la date d'introduction du taureau en liberté avec les femelles au pâturage est le facteur le plus important pour la fécondation des retours.

Enfin, on pourrait craindre que lorsque l'œstrus est induit peu de temps après mise-bas sur des vaches allaitantes, 1'anœstrus de lactation reprenne ensuite. En fait, des essais réalisés en station montrent que lorsque l'on déclenche un œestrus dès 30 jours après la mise-bas sans insémination sur des vaches charolaises allaitantes, tous les animaux reviennent en cestrus normalement.

Dans nos conditions expérimentales, les meilleurs résultats sont obtenus avec les implants SC 2 roog laissés en place pendant Io ou $\mathrm{I} 2$ jours avec injection de $5 \mathrm{mg}$ de valérate d'œestradiol le jour de la pose et sur des races rustiques.

Reçu pour publication en juillet 1973.

\section{REMERCIEMENTS}

Nous remercions les Docteurs Short et Davenport (G. D. Searle et Co., Chicago), ainsi que les Laboratoires Lathevet (Paris) pour la fourniture des implants et les conseils quant à leur utilisation. La mise en place chez les éleveurs n'a été possible que grâce à l'aide apportée par l'Établissement Département d'Élevage du Cantal (MM. Anthony, Rivet, Olivier) et le Centre d'Insémination Artificielle de l'Allier (M. Guezennec).

\section{SUMMARY}

\section{USE OF PROGESTAGENS IN SUB-CUTANEOUS IMPLANTS} TO CONTROL SEXUAL CYCLE OF CATTLE,

Estrus synchronization and related fertility has been tested in cattle when using subcutaneous implants containing norethandrolone or SC 2 I 009 or fluorogestone acetate. The progestagen content of the implant and the time it remains in situ are two important factors.

A lengthening of the treatment duration decreases the rate of cestrus inhibition. For longlasting treatments ( 16 or 18 days), the percentage of cows showing oestrus within four days from implant removal increases with the initial content of the implant : 6 I.5 p. Ioo vs 84.6 p. Ioo with 6 , or $12 \mathrm{mg}$ of SC 2 Ioog, respectively.

Fertility has been studied in a field trial. The progestagen content of the implant has little effect, but lengthening of the treatment duration lowers fertility : 49.4 p. Ioo vs 27.3 p. Ioo for Io and I6-day treatments, respectively, with SC 2 Ioog implants.

Estradiol valerate injection at time of implant insertion increases fertility irrespectively of treatment duration : 45.8 p. Ioo vs $26.9 \mathrm{p}$. Ioo for injected and non-injected cows, respectively.

The use of highly potent progestagens allows for the insertion of small size implants. Crosslink percentage must be studied for each progestagen ( $4.8 \mathrm{p}$. roo for SC 2 Ioog). Among the three progestagens tested only SC 21009 is potent enough to be used in implants.

\section{RÉFÉRENCES BIBLIOGRAPHIQUES}

Bosc M., du Mesnil du Buisson F., Chupin D., ig7o. (Communication personnelle).

Cumming I. A., DE B. Blockey M. A., r97o. The release of luteinizing hormone in ewes following the withdrawal of intravaginal sponges containing a progestagen. Proc. Austr. Soc. Anim. Prod., 8, 383-387. 
Goldenberg R. L., Vattukattis J. L., Ross G., 1972. Estrogen and follicle stimulating hormone interactions on follicle growth in rats. Endocrinology, 90, I492-I 498 .

Hackett A. J., Schechter R. J., Hobson W. C., Hansel W., Ross P. J., 1972. Luteinizing hormone and estrogens during synchronization. J. Anim. Sci, 35, 244 (Abstr.).

Hansel W., 1966. Control of the ovarian cycle in cattle. Proc. 13th Easter School in Agric. Sci., Nottingham, 4I9-443.

Kincl F. A., Rudel H. W., Ig7I. Sustained release hormonal preparations. Acta Endocr., Suppl., 151, 5-30.

Knox J. W., Rabb J. L., Oakes J. Y., Vincent C. K., I972. Progestin injections and ear implants for control of cestrus in cattle. J. Anim. Sci., 34, 354 (Abstr.).

LEMON M., I973. (Communication personnelle).

Liang L., Fosgate O. T., rg7r. Estrus synchronization and subsequent fertility in dairy cattle treated with Nilevar and SC 4640. J. Anim. Sci., 33, 96-98.

Martin E., Espinosa E., Sierra I., Cantalapiedra L., i972. Contrôle du cycle sexuel avec du FGA et de la PMSG chez les bovins de race laitière hollandaise de Santander (Holandesa Santanderina). VII Cong. intern. Reprod. anim. Insem. artif., Munich, 2, 870-873.

Martin E., Espinosa E., Sierra I., Belloc D., ig72. Contrôle du cycle sexuel avec FGA et PMSG chez les vaches de la race Brune (Pardo Suiza) utilisée pour la production de viande. VII Cong. intern. Reprod. anim. Insem. artif., Munich, 2, 893-896.

Mauléon P., Rey J., Mariana J. C., I 968 . Contrôle du cycle ostrien chez la vache à l'aide d'éponges vaginales imprégnées d'acétate de fluorogestone et possibilité d'obtention d'une superovulation limitée. $V I^{\mathrm{e}}$ Cong. intern. Reprod. anim. Insem. antif., Paris, 2, I479-1482.

Pelletier J., Thimonier J., I969. Étude de la décharge ovulante par dosage radioimmunologique de la LH plasmatique chez la brebis normale ou traitée par un progestagene. C. R. Acad. Sc., Paris, Ser. D., 268, 573-576.

Pelletier J., Thimonier J., I973. Influence of fluorogestone acetate on hypothalamo-hypophyseal activity in anostrous dry and lactating ewes. J. Reprod. Fert., 31, 496 (Abstr.).

Priedkalns J., I97I. Effect of melengestrol acetate on the bovine ovary. Z. Zellforsch. Mikrosc. Anat., 122, 85-I IO.

Priedkalns J., I97r. Étude histologique et ultrastructurale de l'ovaire de vache après administration de longue durée de MGA. Bull. Soc. Sci. Vet. Med. Comp., Lyon, 73, 557-576.

Roseman T. J., Higuchi W. I., r970. Release of medroxyprogesterone acetate from a silicone polymer. J. Pharmac. Sci., 59, 353-357.

Wiltbank J. N., Shumway R. P., Parker W. R., Zimmerman D. R., ig67. Duration of œstrus, time of ovulation, and fertilization rate in beef heifers synchronized with dihydroxyprogesterone acetophenide. J. Anim. Sci., 28, 764-767.

Wiltbank J. N., Kasson C. W., I968. Synchronization of cestrus in cattle with an oral progestational agent and an injection of an estrogen. J. Anim. Sci., 27, I I3-I I6.

Wiltbank J. N., Sturges J. C., Wideman D., Lefever D. G., Faulkner L. C., ig7i. Control of cestrus and ovulation using subcutaneous implants and ostrogen in beef cattle. J. Anim. Sci., 33, 600-606.

Wishart D. F., Hoskin B. D., Ig68. Synchronization of ostrus in heifers using intravaginal pessaries impregnated with SC 9880 and PMSG. J. Reprod. Fert., 17, 285-289.

WISHART D. F., I972. Identification of steroids of high activity for control of the œstrous cycle in the dairy heifer. J. Reprod. Fert., 30, 333-334. 